\title{
Decir y no decir lo mismo: acerca de las orientacio- nes y modos de la recontextualización periodística
}

\author{
Sebastián SAYAGO \\ sebastian.sayago@ucv.cl \\ Pontificia Universidad Católica de Valparaíso (Chile)
}

Recibido: 14 de enero de 2014

Aceptado: 11 de julio de 2014

\section{Resumen}

Este trabajo propone un modelo de análisis de la recontextualización discursiva periodística. Para ello, presenta una síntesis de aportes de N. Luhmann (2000), M. Pêcheux $(1978,1990)$ y B. Bernstein (1998) y expone un modelo de análisis de orientaciones y modos elementales de este proceso ideológico-discursivo. Con el fin de demostrar la validez de la propuesta, analiza un caso de recontextualización de la prensa digital chilena del 11 de septiembre de 2012, en ocasión de conmemorarse el $39^{\circ}$ aniversario del golpe militar que derrocara al Presidente Salvador Allende. La metodología utilizada es cualitativa y la técnica de análisis empleada es el análisis del discurso.

Palabras clave: recontextualización, sistema de medios de comunicación de masas, formaciones discursivas, formaciones ideológicas, discurso noticioso.

\section{Say and not say the same: about orientations and modes of the journalistic recontextualization}

\begin{abstract}
This paper proposes an analytical model of news discourse recontextualization. To that aim, it summarizes the contributions made by N. Luhmann (2000), M. Pêcheux $(1978,1990)$ and B. Bernstein (1998) and it presents an analytical model of orientations and elementary modes of this ideological-discursive process. In order to prove the validity of this proposal, a case of Chilean digital media recontextualization is analyzed. This case dates from September 11, 2012, 39 years after the military coup that overthrew President Salvador Allende. A qualitative methodology is used, and discourse analysis is the analytical technique chosen for this study.

Keywords: recontextualization, mass media system, discursive formations, ideological formations, news discourse.

Referencia normalizada

SAYAGO, Sebastián (2015): "Decir y no decir lo mismo: acerca de las orientaciones y modos de la recontextualización periodística". Estudios sobre el Mensaje Periodístico. Vol. 21, Núm. 1 (enero-junio), págs.: 579-597. Madrid, Servicio de Publicaciones de la Universidad Complutense.

Sumario: 1. Introducción. 2. Sistemas, formaciones y recontextualización. 3. Orientaciones y modos de la recontextualización periodística. 4. Metodología y corpus. 5. Análisis; 5.1. Contextualización del caso: las FDs de la prensa chilena; 5.2. La fuente: el discurso a recontextualizar; 5.3. Las recontextualizaciones contraorientadas; 5.3.1. La Tercera y La Cuarta; 5.3.2. El Mercurio y La Segunda; 5.4. Las recontextualizaciones coorientadas; 5.4.1. The Clinic; 5.4.2. El Mostrador. 6. Conclusiones. 7. Referencias bibliográficas. 8. Corpus de publicaciones digitales.
\end{abstract}

\section{Introducción}

En el presente trabajo, seguimos dos objetivos:

a) esbozar una propuesta sistémica de la recontextualización discursiva periodística,

b) presentar y aplicar un modelo de orientaciones y modos de esta recontextualización. 
Para cumplir el primer objetivo, proponemos una síntesis de tres teorías:

- la teoría del funcionamiento sistémico de los medios de comunicación de Niklas Luhmann (2000),

- la teoría materialista del discurso de Michel Pêcheux (1978, 1990), y

- la teoría de los campos recontextualizadores de Basil Bernstein (1998).

Para alcanzar el segundo objetivo, desarrollamos y ejemplificamos una propuesta de reconocimiento de las orientaciones y modos elementales de la recontextualización discursiva perodística.

El trabajo se estructura en dos partes. En la primera, exponemos los lineamientos conceptuales utilizados. En la segunda, realizamos un estudio de caso. Se trata de un proceso de recontextualización de la prensa digital chilena efectuado el 11 de septiembre de 2012, en ocasión de conmemorarse el $39^{\circ}$ aniversario del golpe militar que derrocara al Presidente Salvador Allende.

\section{Sistemas, formaciones y recontextualización}

Las propuestas teóricas de N. Luhmann, M. Pêcheux y B. Bernstein se sustentan sobre el modelo teórico paradigmático: el sistema (Ladrière, 1978: 37-38). Este es un esquema representacional que atribuye a las entidades algunos rasgos básicos: regularidad, permanencia en el tiempo, variación, límites, procesos internos, posibilidades de cambio, etc.

De los tres autores, es Luhmann quien explotó este concepto con mayor detalle y con mayor grado de explicitud. En Pêcheux y Bernstein, en cambio, el sistema es tomado como un supuesto que posibilita la estructuración de diferentes nociones: formaciones ideológicas y discursivas (Pêcheux, 1978, 1990), dispositivos y campos (Bernstein, 1998). Además de compartir esta perspectiva sistémica, los tres conciben la comunicación como fundamento de interacción y de organización social. Consideramos que es posible componer una propuesta sistémica de recontextualización periodística del discurso mediante la síntesis de aportes de estos tres teóricos.

En primer lugar, asumimos que el sistema de medios de comunicación de masas (en adelante, SMCM) se encarga de la observar la sociedad y de producir y reproducir inseguridades acerca del futuro (Luhmann, 2000: 50-55). Para llevar adelante esta observación de segundo orden, filtra los acontecimientos que percibe mediante la aplicación del código informable/no informable y despliega un discurso noticioso que adopta dos grandes modalidades genéricas: las noticias y los reportajes.

Luhmann afirma que los sistemas sociales (y el SMCM es uno de ellos) existen en un universo de sentido, elaborado heterorreferencialmente y autorreferencialmente, el cual, lejos de ser un simple almacén de representaciones, es un proceso de diferenciación entre lo que hay que recordar y lo que hay que olvidar (Luhmann, 1998: 78112; 2000: 57-58; 2006: 36-37). Si aceptamos que este universo de sentido no es neutro, puesto que es el resultado de procesos históricos de dominación y de resistencias, de aproximaciones y de distanciamientos, encontramos aquí un punto de articulación con la teoría materialista del discurso de Pêcheux. El universo de sentido en el que opera el SMCM puede ser concebido como una formación ideológica (FI) y sus modalidades comunicativas, formaciones discursivas (FDs). 
Así como Pecheux (1978: 235), en el interior de la FI religiosa medieval, distinguió dos FDs (la alta clerecía y la baja clerecía), es posible distinguir diferentes FDs en el interior de la FI del SMCM. Para ello, recurriendo a criterios de distinción usuales en el campo de investigación de la comunicación social, podemos caracterizar los discursos de acuerdo con la ubicación que asignemos a los medios que los producen. Podríamos distinguir: medios conservadores vs. medios críticos (o progresistas), medios oficialistas vs. medios no oficialistas (u opositores), medios populares vs. medios elitistas, medios de izquierda vs. medios de derecha, etc. La lista de las distinciones no es exhaustiva y cada una de ellas puede ser reespecificada en una escala de gradualidad.

Las diferentes FIs y FDs de una formación social conforman un mosaico complejo e inestable, puesto que hay relaciones multidireccionales que provocan desfasajes, rupturas y desplazamientos. Vayamos al caso de la FI de la prensa, el subsistema del SMCM del que nos ocupamos en este trabajo.

Por un lado, la FI provee a las FDs de matrices de sentido, acotando el haz de dispersión de lo representable. De este modo, hay un primer umbral de noticiabilidad. Por otro lado, las diferentes FDs dialogan entre sí, de un modo más o menos directo, al cotejar temas y formas de tratamiento, lo que puede dar lugar a la inclusión de temas que tensionen los límites de la FI. Estos vínculos de préstamos y referencias son propiciados por las reglas de competencia comercial y favorecidos, en la actualidad, por las NTICs, lo que incrementa la redundancia en la información producida, recontextualizada y puesta en circulación por el SMCM. Así, las FDs establecen relaciones de semejanza y de diferenciación entre sí y los cambios que ocurran en unas pueden promover cambios en las otras.

Pecheux denomina interdiscurso al punto de exterioridad que rodea, presiona y constituye cada FD. Es todo eso que se dice en otros lados, que también se podría decir en el interior de cada FD y que, por eso mismo, obliga a la realización de operaciones de filtro y de exclusión. El interdiscurso se revela como lo preconstruido, como eso-que-está-ahi y que todos saben o pueden saber, sin que lo comuniquen.

Las distinciones hechas hasta aquí permiten reconceptualizar la idea de recontextualización discursiva expuesta por Bernstein principalmente en su último libro, $\mathrm{Pe}$ dagogía, control simbólico e identidad (1998). Si bien su planteo apunta a la explicación de la naturaleza del discurso pedagógico, hay muchas nociones que pueden ser fructíferas para el análisis de otros discursos sociales. Tal es el caso del discurso noticioso, el cual, de modo similar al discurso pedagógico, también actúa como un principio recontextualizador que establece y reproduce categorías, jerarquías, grados de relevancia y modos de desarrollo (más o menos exhaustivos, más o menos explicativos, más o menos objetivos).

Sin embargo, la recontextualización periodística exhibe un rasgo que Bernstein no resalta en la recontextualización pedagógica: la orientación. Con esta noción nos referimos al tratamiento de refuerzo o de resistencia que cada empresa o cada grupo periodístico pueden dar a la representación de un acontecimiento generado en el exterior del sistema de medios o tratado por otro grupo integrante del sistema de medios. 


\section{Orientaciones y modos de la recontextualización periodística}

En el modelo bernsteniano de recontextualización, podemos señalar dos aspectos significativos de acuerdo con los objetivos de nuestro trabajo. El primero es el de la direccionalidad y el segundo el de la relación entre la ideología y el discurso.

Para explicar la estructura del dispositivo pedagógico, Bernstein propone un orden jerárquico y lineal: las novedades comienzan en una instancia (la de la producción de la teoría) y, luego, son apropiadas en una instancia atravesada por las acciones y objetivos del Estado y del mercado (el campo recontextualizador pedagógico). En el caso de la prensa, el origen de los discursos es multipolar, ya que actúan como fuente actores de diferentes sistemas sociales (el político, el económico, el artístico, el deportivo, el religioso, el judicial, etc.). Incluso la misma prensa suele construir una fuente interna del discurso, cuando, por ejemplo, realiza entrevistas o cuando publica editoriales o columnas de opinión.

El segundo aspecto a señalar es la relación entre lo ideológico y lo discurso. Según Bernstein, el dispositivo pedagógico involucra tres clases de reglas: las ditributivas, las recontextualizadoras y las evaluadoras. Dejamos de lado las últimas, ya que tienen validez para la comunicación en el contexto del aula y nos referiremos a las otras dos, que son más relevantes para el análisis del discurso de la prensa. Las reglas distributivas "especializan el acceso a los campos en los que puede producirse legítimamente nuevo conocimiento" (Bernstein, 1998: 144) y las recontextualizadoras "regulan el trabajo de los especialistas en el campo recontextualizador los cuales construye el 'qué' y el ‘cómo' del discurso pedagógico" (1998: 144). Ambas especifican la diferencia entre lo pensable y lo decible. Cada campo produce el discurso que recontextualiza el conjunto de saberes que le es asignado según la división social del conocimiento.

Si asumimos que cada campo o formación discursiva tiene reglas, objetivos y archivos particulares, es posible explicar y anticipar tendencias en los procedimientos de recontextualización. La representación discursiva de un mismo acontecimiento puede ser reforzada en un medio y cuestionada en otro. En el primer caso, hablaremos de recontextualización positiva o coorientación; en el segundo, de recontextualización negativa o contraorientación. Estas son las dos orientaciones elementales de la recontextualización discursiva: cada medio puede estar más o menos a favor del discurso que recontextualiza (y de las representaciones que este materializa) o más o menos en contra. El tratamiento objetivo y neutral es excepcional.

A su vez, cada orientación puede presentar dos modos, dependiendo del grado de manifestación de la aceptación o del rechazo del discurso recontextualizado. Diremos que una orientación dada es fuerte o exhibe un modo fuerte cuando hay marcas discursivas que explicitan enfáticamente la postura positiva o negativa del medio. El modo será débil, si las marcas discursivas son poco explícitas.

Vale apuntar que los discursos recontextualizados no son neutros. Portan marcas ideológicas de diversas orientaciones e intensidades, la cuales pueden ser filtradas o no en el proceso de recontextualización. Así, se abre un abanico de posibilidades. Por ejemplo, cuando un discurso está notoriamente marcado y la recontextualización conserva esas marcas, diremos que la recontextualización es débil (porque no hay trans- 
formaciones discursivas) y coorientada (porque demuestra afinidad ideológica). Cuando, en cambio, un discurso notoriamente marcado es sometido a un proceso de transformación que altera las marcas de una manera clara, diremos que la recontextualización es fuerte, pudiendo además haber coorientación o contraorientación. Como veremos más adelante, hay que tener en cuenta también que tanto la orientación como el modo son aspectos que trascienden las meras formas lingüísticas y se vinculan con la memoria discursiva de cada medio.

\section{Metodología y corpus}

Para ejemplificar la productividad del enfoque teórico propuesto, analizamos un caso particular: el tratamiento dado por los principales medios de prensa digital de Chile a una discusión suscitada en el Congreso de la Nación, el 11 de septiembre de 2012. El episodio ocurrió en la mañana de ese día, cuando un diputado independiente, René Alinco, solicitó que se hiciera un minuto de silencio en homenaje a Salvador Allende y algunos diputados oficialistas expresaron su rechazo.

El caso cumple con tres criterios básicos: relevancia social, pertinencia teórica y adecuación empírica.

- Relevancia social. Es un acontecimiento cuyo tratamiento noticiable es una clara irritación de la moral de la sociedad (Luhmann, 2000), en tanto plantea una cuestión todavía conflictiva en la sociedad chilena: la relación entre democracia y dictadura militar.

- Pertinencia teórica. La conflictividad ideológica de la noticia produce una clara separación de aguas entre las formaciones discursivas de la prensa, lo que permite apreciar las diferentes operaciones ideológicas y discursivas en el proceso de recontextualización efectuado por cada una.

- Adecuación empírica. El tratamiento periodístico puede ser estudiado mediante el análisis de un corpus acotado, constituido por la serie de textos noticiosos (crónicas) que, sobre este tema, publicaron los principales medios chilenos ese día: El Mercurio, La Tercera, La Cuarta, The Clinic y El Mostrador.

La metodología utilizada es cualitativa, en tanto la investigación es un trabajo de descripción, interpretación y explicación de un caso particular, sin recurso a la cuantificación de los datos. La técnica de análisis empleada es el análisis del discurso.

Todos los textos analizados, en su versión completa, están indicados en la sección 7. Referencias bibliográficas.

\section{Análisis}

\subsection{Contextualización del caso: las FDs de la prensa chilena}

De acuerdo con los objetivos de este trabajo y con la naturaleza del caso seleccionado, adoptamos como criterio diferenciador de las FDs su posicionamiento ideológico en un espectro que establece dos regiones: derecha e izquierda. En Chile, esta categorización es utilizada por líderes políticos, medios y ciudadanos para diferenciar, por un lado, a quienes adhieren a una economía neoliberal, valoran el alineamiento internacional con Estados Unidos y justifican el autoritarismo del gobierno 
militar de Augusto Pinochet y, por otro, a quienes adhieren a una economía estatista, valoran el alineamiento internacional con países de tendencia socialista y reprueban el golpe militar que acabó con el gobierno democrático de Salvador Allende (Dávila y Fuentes, 2003; Alcántara Sáez, 2004; Luna y Zechmeister, 2010; Avendaño, 2011). La diferenciación derecha-izquierda también es utilizada para caracterizar los medios de comunicación (Sunkel y Geoffroy, 2001; Mönckeberg, 2009; Castillo, 2011).

Como todo sistema social, el SMCM es producto de una historia y exhibe de diferentes maneras las huellas de las tensiones y asimetrías de la formación social. En Chile, este sistema se caracteriza por una gran concentración de la propiedad de los medios, sobre todo en la prensa impresa tradicional. Hay un duopolio conformado por El Mercurio y COPESA, que fue creado a la sombra de la dictadura militar de Augusto Pinochet (Dermota, 2002; Corrales Jorquera y Sandoval Moya, 2005; Corrales Jorquera y Sandoval Moya, 2005; González Rodríguez, 2008; Merrill, 2010). El Mercurio es un conglomerado de medios, cuyo producto más importante es el diario homónimo. COPESA también produce una variedad de publicaciones, entre ellas, los diarios que se ubican en segundo y tercer lugar de ventas luego de El Mercurio: La Tercera y La Cuarta.

Resultado de una alianza entre militares y sectores civiles, este duopolio ejerció el control de la formación ideológica de la prensa chilena, promoviendo la difusión de un conservadurismo cultural y político y un modelo económico neoliberal. La dictadura pinochetista sostuvo esta modificación del sistema de medios utilizando como principales mecanismos la clausura de la prensa no alineada, la persecución (e incluso el asesinato) de periodistas considerados peligrosos y el otorgamiento de grandes ventajas financieras a ambos grupos.

Durante gran parte del gobierno militar y aun durante casi toda la primera década de democracia, el duopolio estableció el discurso dominante (Raiter, 2008: 19-23), es decir, el eje de referencias que todas las formaciones discursivas de la prensa debían observar para producir un discurso noticioso válido. En otras palabras, el duopolio consiguió el "monopolio ideológico" (Corrales Jorquera y Sandoval Moya, 2005: 19).

Esa fue la situación, hasta que se produjeron cambios que indicaban rupturas discursivas, asociadas también a rupturas en la formación ideológica del SMCM. En 1998, apareció en escena la revista Rocinante, una revista de crítica política y cultural. Un año después, comenzó a circular The Clinic. En el 2000, empezó a publicarse El Mostrador, el primer diario exclusivamente digital de Chile, posicionado en un espacio de centroizquierda. Al poco tiempo, entraron en escena las revistas El Periodista (2001), Plan B (2003) y el Diario Siete (2005). La estructura de un avisaje asociado al monopolio ideológico y el rol del Estado como sostén económico del duopolio mediante la publicidad, entre otros factores, determinaron que la mayoría de la mayoría de esos nuevos medios deban cerrar sus puertas, pese a la calidad periodística que muchos les reconocieron. Al día de hoy, subsisten The Clinic, El Mostrador y El Periodista.

Actualmente, hay dos formaciones discursivas dentro del sistema de la prensa chilena: una, posicionada en la derecha, y otra, posicionada en la izquierda. En la formación discursiva de derecha, se trata con seriedad el establishment político (sobre todo, 
al de centroderecha y derecha). Los medios destinados a los sectores populares simulan un tratamiento despolitizado de la actualidad mediante dos recursos: la exclusión de noticias políticas de la Agenda (salvo que el interdiscurso las vuelva inevitables) y la presuposición de un orden político y económico que no merece ser criticado. En la formación discursiva de izquierda, se representan de manera negativa las principales fuerzas políticas: la alianza oficialista de derecha-centroderecha integrada por Unión Demócrata Independiente (UDI) y Renovación Nacional (RN), por un lado, y, por otro, la alianza opositora de centroizquierda conformada por democratacristianos, socialistas y comunistas. Además, se reivindica permanentemente la figura de Salvador Allende y se responsabiliza a Estados Unidos como promotor del golpe de Estado de 1973, con la complicidad de las fuerzas armadas y la clase alta chilena.

En la formación discursiva de derecha, se ubican los diarios El Mercurio, La Segunda (del mismo grupo), La Tercera y La Cuarta, entre otras publicaciones. En la formación discursiva de izquierda, las revistas The Clinic, El Periodista y El Mostrador. Salvo este último, todos los demás medios ofrecen su versión en papel y su versión digital con actualizaciones permanentes.

\subsection{La fuente: el discurso a recontextualizar}

El primer medio de prensa digital que dio a conocer el episodio ocurrido el martes 11 de septiembre de 2012 en la Cámara de Diputados fue la radio Cooperativa, un medio ubicado normalmente en el centro del eje derecha-izquierda (Castillo, 2011), vinculado al partido democratacristiano. En su página web, a las 12:53 de ese día, publicó el siguiente texto noticioso (TN):

\section{TN de radio Cooperativa}

Título: Diputado UDI Ignacio Urrutia calificó a Allende como "el cobarde que se suicidó"

Bajada: El parlamentario gremialista se enfrascó en una disputa con sus pares opositores. Éstos habían pedido un minuto de silencio por las víctimas de la dictadura.

Cuerpo de la noticia:

Un polémico episodio protagonizó este martes el diputado UDI Ignacio Urrutia al insultar al ex Presidente Salvador Allende en medio de la conmemoración de los 39 años del golpe de Estado en el Congreso, refiriéndose al líder socialista como "el cobarde que se suicidó".

Las expresiones de Urrutia se produjeron luego de que el diputado por Aysén René Alinco solicitara realizar un minuto de silencio al inicio de la sesión en la Cámara Baja, en recuerdo de las víctimas de la dictadura y del fallecido Presidente Allende.

“¿A los cobardes que se suicidaron ese día también le vamos a rendir homenaje? ¿Al cobarde que se suicidó ese día también? No puedo creerlo, presidente", lanzó Urrutia dirigiéndose al presidente de la Cámara de Diputados, el RN Nicolás Monckeberg, que en medio de la tensión en el hemiciclo accedió a la petición de Alinco.

Ignacio Urrutia Bonilla es diputado desde el año 2002 y representa en la Cámara Baja al Distrito N40, que comprende las comunas de Cauquenes, Chanco, Longaví, Parral, Pelluhue y Retiro, en la Región del Maule. 
Este texto noticioso (TN) materializó patrones ideológicos y discursivos que condicionaron las sucesivas recontextualizaciones. Veamos sus características más sobresalientes:

1. Titular. El TN de radio Cooperativa llevaba por título: Diputado UDI Ignacio Urrutia calificó a Allende como "el cobarde que se suicidó". Proponía como el hecho noticioso más relevante la valoración negativa del ex Presidente hecha por un diputado particular del gobierno. La bajada indica que Urrutia fue el responsable de una disputa con un grupo indeterminado de "pares opositores", quienes habían pedido un minuto de silencio por "las víctimas de la dictadura".

2. Organización de la información. Las tres partes del cuerpo de la noticia (introducción, desarrollo y cierre) presentó la información de la siguiente manera:

- Entrada o lead: definición de la situación como un "polémico episodio" protagonizado por Urrutia.

- Desarrollo: narración sucinta de la secuencia pedido de Alinco - reacción de Urrutia.

- Cierre: Mención de datos que permitan identificar a Urrutia (año desde el que ejerce el cargo de diputado y el distrito y las comunas que representa).

Se incluía debajo del cuerpo principal una foto de Urrutia, con un pie que hacía mencionaba el repudio que el "exabrupto del legislador" había recibido a través de las redes sociales.

3. Estilo. El estilo utilizado es formal y las selecciones léxicas ponen de manifiesto un rechazo a la conducta de Urrutia, a la que se referían mediante la construcción verbal "insultar al ex Presidente Allende". Se usaron también las expresiones "golpe de Estado" y "recuerdo de las víctimas de la dictadura y del fallecido Presidente Allende".

El TN de Cooperativa siguió una matriz de sentido que tiene una validez general dentro y afuera de la formación ideológica de la prensa. Podríamos formularla así: No se debe criticar públicamente a los hombres que tienen X cualidad y podríamos especificar la cualidad $\mathrm{X}$ en uno de los siguientes rasgos o en la combinación de todos: el mérito de haber dado la vida por sus ideales, el mérito de haber sido mandatarios/funcionarios honestos, el mérito de haber sido presidente democrático de la nación. Para sistematizar el modo como se materializó discursivamente la matriz, vamos a recomponer las principales cláusulas de la primera parte del TN: el título, la bajada y la entrada (primer párrafo del cuerpo de la noticia). ${ }^{1}$

Tabla 1. Principales cláusulas del TN de radio Cooperativa

\begin{tabular}{|c|l|l|l|}
\hline $\begin{array}{c}\text { Ubicación de } \\
\text { la cláusula }\end{array}$ & \multicolumn{1}{|c|}{ Actor } & \multicolumn{1}{|c|}{ Acción } & Circunstancias \\
\hline Título & $\begin{array}{l}\text { Diputado UDI } \\
\text { Ignacio Urrutia }\end{array}$ & $\begin{array}{l}\text { calificó a Allende como } \\
\text { "el cobarde que se } \\
\text { suicidó" }\end{array}$ & \\
\hline Bajada & $\begin{array}{l}\text { El } \\
\text { parlamentario } \\
\text { gremialista }\end{array}$ & $\begin{array}{l}\text { se enfrascó en una } \\
\text { disputa con sus pares } \\
\text { opositores }\end{array}$ & \\
\hline
\end{tabular}

1 Reconoceremos solo tres constituyentes en las cláusulas: actor, acción y circunstancias. El análisis propuesto no requiere una distinción más pormenorizada. 


\begin{tabular}{|c|c|c|c|}
\hline \multirow{3}{*}{ Entrada } & \multirow{3}{*}{$\begin{array}{l}\text { El diputado } \\
\text { UDI Ignacio } \\
\text { Urrutia }\end{array}$} & $\begin{array}{l}\text { protagonizó un polémico } \\
\text { episodio }\end{array}$ & este martes \\
\hline & & $\begin{array}{l}\text { (al) insultar al ex } \\
\text { Presidente Salvador } \\
\text { Allende }\end{array}$ & $\begin{array}{l}\text { en medio de la } \\
\text { conmemoración de los } 39 \\
\text { años del golpe de Estado en } \\
\text { el Congreso }\end{array}$ \\
\hline & & $\begin{array}{l}\text { refiriéndose al líder } \\
\text { socialista como "el } \\
\text { cobarde que se suicidó" }\end{array}$ & \\
\hline
\end{tabular}

Las cláusulas guardan entre sí una relación de paráfrasis: dicen más o menos lo mismo. En el título, la bajada y la entrada se destacaba a un solo actor, el diputado Urrutia, al que se mencionó tres veces, con énfasis en su rol institucional y en su identificación partidaria ("diputado UDI", "parlamentario gremialista") y del que se predicaron cinco veces dos acciones negativas (insultar a un ex presidente e iniciar un incidente).

Las equivalencias parafrásticas generaron un efecto de redundancia que garantizó la novedad, la relevancia y la verosimilitud del acontecimiento representado. Se repitió la información porque se la presentaba como novedosa, porque se la presumía relevante y porque reforzaba el patrón de verosimilitud del mundo posible construido por el discurso del medio.

Este TN estableció un umbral de noticiabilidad del acontecimiento que obligó a otros medios a realizar una inmediata recontextualización. Como era previsible, cada FD realizó operaciones ideológicas y discursivas particulares.

\subsection{Las recontextualizaciones contraorientadas}

En la FD de derecha, se efectuaron recontextualizaciones controrientadas. Seleccionaremos las dos más importantes, una corresponde al grupo COPESA y la otra, al grupo El Mercurio.

\subsubsection{La Tercera y La Cuarta}

Las primeras operaciones de apropiación y recontextualización fueron realizadas por el grupo COPESA, a través de sus diarios La Tercera y La Cuarta. Este proceso se materializó en dos momentos puntuales: a las 13:14 y a las 21:21, cuando La Tercera y La Cuarta publicaron la noticia, respectivamente.

Respecto del TN de radio Cooperativa, La Tercera mantenía la representación del protagonismo de Urrutia, al que sumaba la participación de Iván Moreira, otro diputado UDI que objetó el pedido de minuto de silencio.

1. Titular. El texto se titulaba: Diputados UDI protagonizan incidente en la Cámara en minuto de silencio por Allende y los fallecidos del 11 de septiembre. En la bajada se mencionaba a dos diputados oficialistas, Ignacio Urrutia e Iván Moreira, cuya acción es recategorizada como la manifestación en contra de una iniciativa.

2 En Chile, gremialista es un adjetivo con el que también se hace referencia a los miembros de la UDI. 
2. Organización de la información. Este aspecto mantenía el patrón del texto de radio Cooperativa:

- Entrada o lead: definición de la situación.

- Desarrollo: narración sucinta de la secuencia pedido de Alinco - reacciones de diputados oficialistas.

- Cierre: Reiteración del marco y mención de la "fuerte resonancia en las redes sociales".

3. Estilo. Los principales cambios estilísticos se produjeron en el nivel léxico, con supresiones o sustituciones orientadas a la disminución de la gravedad del hecho y mitigar la presentación negativa tanto de los diputados oficialistas como del golpe de Estado. Veamos los principales cambios:

a. Se sustituyó la expresión "polémico episodio" por dos expresiones nominales: "incidente" y "polémica".

b. Se incorporaron más diputados oficialistas como responsables de las quejas. A Ignacio Urrutia, se sumaron Iván Moreira (UDI) y René Manuel García (RN)

c. No se mencionó que hubo un insulto a Salvador Allende.

d. Se cambió la $P$ mayúscula por la minúscula en la denominación de Allende: "ex presidente". Este dato podría no ser significativo, ya que muchos diarios no utilizan las mayúsculas para referenciar los cargos de los funcionarios públicos. Pero no es el caso de La Tercera.

e. En el cuerpo de la noticia, sustituyó las expresiones "golpe de Estado" y "dictadura" por "intervención militar", "ese período" y "régimen militar". También sustituyó la expresión "víctimas" por "fallecidos".

f. Estableció una relación de sucesión temporal y no de causa-efecto entre la "intervención militar" y los "fallecidos".

Horas más tarde, casi sobre el cierre de la jornada periodística, la presión del interdiscurso obligó a La Cuarta, el diario popular de COPESA, a romper su criterio de exclusión de noticias políticas. La Tercera había publicado el TN en la sección Política. Como La Cuarta no tiene esta sección, tuvo que incluirlo en Crónica, una categoría general. Este TN fue una reelaboración del TN publicado por La Tercera, el que, a su vez, fue una reelaboración del TN publicado por radio Cooperativa.

1. Titular. El título elegido fue: Minuto de silencio a Allende prendió la mecha en el Congreso. Podemos reconocer en él una doble metáfora:

a. El minuto de silencio a Salvador Allende hizo algo.

b. Algo prendió la mecha.

De manera implícita, la primera metáfora responsabilizaba del bochorno a los responsables del pedido del minuto de silencio. La segunda completaba el sentido negativo, apelando a una imagen común en la prensa sensacionalista: la de la explosión desencadenada por un hecho imprevisto y escandaloso. En este caso, ese hecho es "el minuto de silencio a Salvador Allende". Así, se reafirmaba el encuadre del diario según el cual la política es algo caótico y nocivo, por culpa de los sectores de izquierda (Sunkel, 2002: 64-68). La idea de la explosión de una bomba en el Congreso activó, de modo más o menos implícito, sentidos específicos (atentado a la democracia, alteración del orden, regreso a la violencia del pasado, etc.). La bajada fue un resumen del 
segundo párrafo del TN de La Tercera, el cual comenzaba con el sustantivo "polémica", derivado del adjetivo constituyente de la expresión "polémico episodio", formulada en el TN de Cooperativa. Se decía: Polémica se originó luego de que el Diputado René Alinco solicitara un momento para recordar al ex presidente. Ya no había más responsable que Alinco.

2. Organización de la información. Al igual que La Tercera, este diario mantuvo la organización del $\mathrm{TN}$ de radio Cooperativa.

- Entrada: definición de la situación de un modo cómico, insistiendo en el pedido de minuto de silencio como la causa del "cuático [desopilante, ridículo] incidente".

- Desarrollo: narración sucinta de la secuencia pedido de Alinco - reacciones de diputados oficialistas.

- Cierre: reemplazo de la expresión "usuarios" (de las redes sociales) de La Tercera por "ciberñoños", connotando de manera peyorativa que quienes dieron importancia a este asunto son personas ingenuas y adictas a las redes sociales.

3. Estilo. La Cuarta ridiculizó la conducta de todos los diputados involucrados en la discusión, poniendo en un mismo nivel tanto a quien propuso rendir un homenaje al ex presidente fallecido como a quienes reaccionaron de manera insultante, defendiendo el golpe militar.

La recontextualización mantuvo la matriz de sentido de La Tercera y redefinió el eje de referencias utilizando su característico estilo, el cual combina los registros informal y formal. Con el primero, logra la distancia ridiculizante; con el otro, logra un sesgo protocolar que normaliza la realidad construida. Mantiene todos las sustituciones léxicas hechas por La Tercera ("intervención militar" por "golpe de Estado", "fallecidos" por "víctimas", etc.). Respecto del TN del otro diario de la empresa, la recontextualización fue coorientada débil, lo que no hizo más que reforzar la recontextualización contraorientada fuerte del discurso noticioso de COPESA en relación con el discurso de radio Cooperativa y el de todos los discursos evocados y presupuestos por este. En otras palabras, el grupo empresarial no quería deslegitimar solo el discurso de un medio de prensa, sino toda una línea de ideológica y discursiva que condena la dictadura militar de Pinochet y que critica a los funcionarios que la han apoyado.

En el TN de La Tercera, no hay cláusulas referidas a diputados de la oposición u otros actores. En el TN de La Cuarta, en cambio, no hay cláusulas referidas a diputados oficialistas. A continuación, ordenamos las principales cláusulas de los dos diarios:

Tabla 2. Principales cláusulas de los TNs de los diarios La Tercera y La Cuarta

\begin{tabular}{|c|c|c|c|c|}
\hline Diario & $\begin{array}{l}\text { Ubicación de } \\
\text { la cláusula }\end{array}$ & Actor & Acción & Circunstancias \\
\hline \multirow{3}{*}{ La Tercera } & \multirow[b]{2}{*}{ Título } & \multirow[b]{2}{*}{ Diputados UDI } & \multirow[b]{2}{*}{ protagonizan incidente } & en la Cámara \\
\hline & & & & $\begin{array}{l}\text { en minuto de silencio por } \\
\text { Allende y los fallecidos del } 11 \\
\text { de septiembre }\end{array}$ \\
\hline & Bajada & $\begin{array}{l}\text { Iván Moreira e Ignacio } \\
\text { Urrutia }\end{array}$ & $\begin{array}{l}\text { se manifestaron en contra } \\
\text { de la iniciativa de la } \\
\text { Cámara de Diputados } \\
\text { efectuada esta mañana }\end{array}$ & $\begin{array}{l}\text { en el marco de una nueva } \\
\text { conmemoración del golpe de } \\
\text { Estado de } 1973\end{array}$ \\
\hline
\end{tabular}




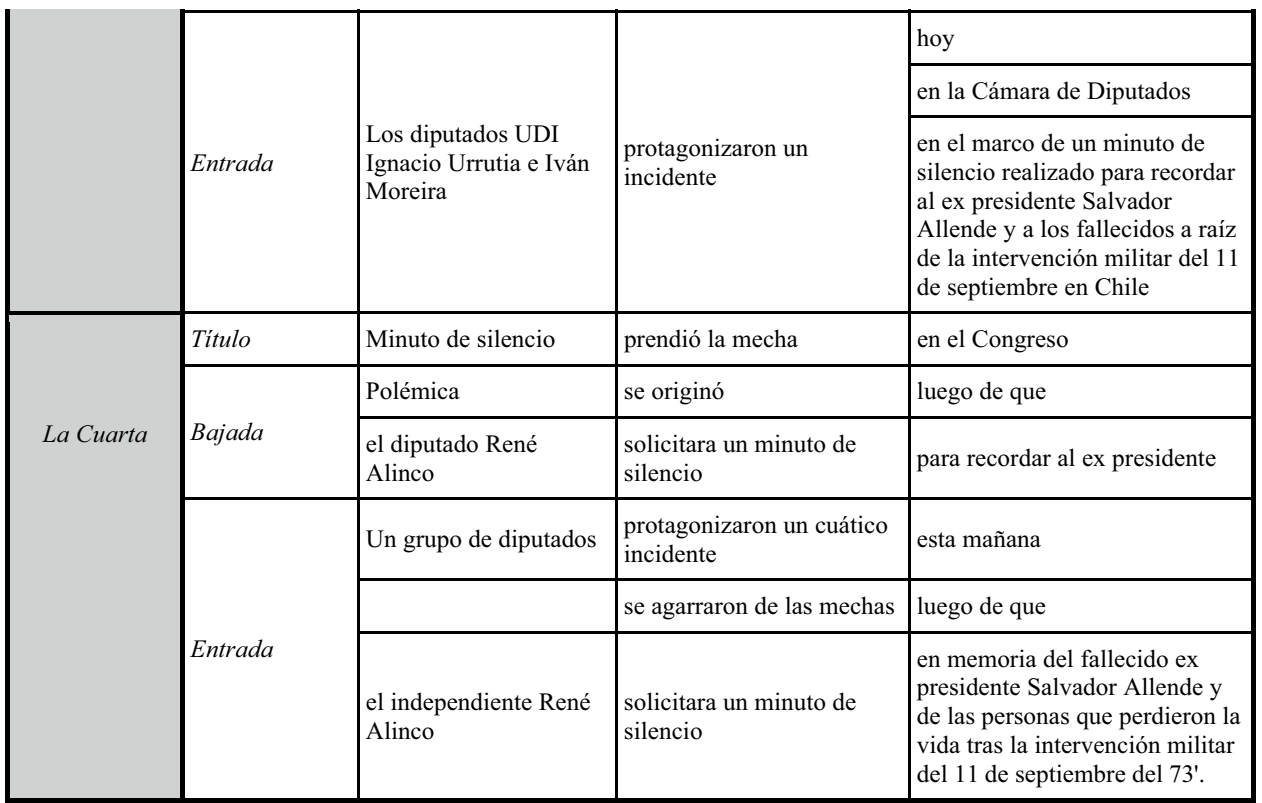

La lectura de la Tabla 2 hace evidente el desplazamiento de sentido promovido por el discurso de COPESA: pasó de la mitigación de la responsabilidad del diputado Urrutia a la responsabilización del diputado Alinco y a la banalización del acontecimiento.

Mientras en el TN de La Tercera los únicos actores representados en las cláusulas analizadas fueron los diputados de la UDI, en el TN de La Cuarta hay un énfasis diferente y una mayor dispersión. La única repetición se produce en una cláusula subordinada de la bajada y en una cláusula subordinada de la entrada, es decir, en una zona estructuralmente importante porque expresa la información temática. La repetición enfatizó la solicitud de Alinco como causa del incidente.

En este TN, el juego de relaciones equivalentes y de desplazamientos sintácticos produjo un efecto de desorden: se hablaba del "minuto de silencio", de la "polémica", de "el diputado René Alinco", de un "grupo de diputados". Sin embargo, en esta dispersión, subyace un patrón de coherencia: había campos semánticos definidos cuyos elementos constitutivos aparecieron en diferentes posiciones. Así, por ejemplo, el concepto de escándalo fue trabajado parafrásticamente como actor ("polémica") y como acción ("prendió la mecha", "protagonizaron un cuático incidente" y "se agarraron de las mechas").

Hay también un recurso de expansión en circunstanciales de la bajada y de la entrada: "para recordar al ex presidente" / "en memoria del fallecido ex presidente Salvador Allende y de las personas que perdieron la vida tras la intervención militar del 11 de septiembre del 73"'. Se podría objetar que este recurso sea ideológicamente significativo, ya que es previsible que la entrada retome y amplíe la información dada en la bajada. Sin embargo, se trata de un desarrollo marcado ideológicamente: es utilizado para cancelar la idea de golpe de Estado y de la existencia de víctimas. 
Las relaciones parafrásticas expresadas por las cláusulas del grupo COPESA expresaron una colisión entre dos matrices de sentido. Una es la matriz ya señalada: No se debe criticar públicamente a los hombres que tienen X cualidad. La otra es la matriz predominante en la categoría política: El gobierno debe administrar bien el Estado. Hay una colisión porque, por un lado, en el discurso de los dos diarios, se admite que no está bien calificar de "cobarde" a un ex presidente, pero, por otro, se trata de un ex presidente al que históricamente el discurso de ambos diarios ha construido como alguien que administró mal el Estado, razón por la cual los militares se vieron obligados a intervenir.

Este fenómeno nos demuestra que las matrices de sentido no son abstracciones vacías de valor ideológico, ya que son especificados en ejes de referencialidad determinados. Así, Salvador Allende no es solo un nombre propio, sino un concepto trabajado históricamente en un contexto discursivo particular.

Vemos, en definitiva, que el discurso de Cooperativa fue sometido a una recontextualización contraorientada fuerte en los dos diarios del grupo COPESA.

\subsubsection{El Mercurio y La Segunda}

La recontextualización efectuada por el grupo El Mercurio es interesante, porque mantuvo los patrones estilísticos del texto de Cooperativa. Con el título Diputado UDI lanzó insulto contra Allende en el Congreso, el TN publicado a las 15:02 en El Mercurio fue reproducido poco después, literalmente, en La Segunda. No se realizaron sustituciones: se utilizaron las expresiones "víctimas de la dictadura", "golpe de Estado" y "ex Presidente Salvador Allende" con $P$ mayúscula. Se calificó la conducta de Urrutia como un insulto.

Veamos las principales cláusulas de este TN:

Tabla 3. Principales cláusulas de los TNs de los diarios El Mercurio y La Segunda

\begin{tabular}{|c|c|c|c|}
\hline $\begin{array}{l}\text { Ubicación de la } \\
\text { cláusula }\end{array}$ & Actor & Acción & Circunstancias \\
\hline Título & Diputado UDI & lanzó insulto contra Allende & en el Congreso \\
\hline Bajada & $\begin{array}{l}\text { El diputado Ignacio } \\
\text { Urrutia (UDI) }\end{array}$ & $\begin{array}{l}\text { manifestó: “¿A los cobardes } \\
\text { que se suicidaron ese día } \\
\text { también le vamos a rendir } \\
\text { homenaje?” }\end{array}$ & $\begin{array}{l}\text { durante un minuto de silencio en } \\
\text { recuerdo de las víctimas de las } \\
\text { violaciones de los derechos } \\
\text { humanos }\end{array}$ \\
\hline \multirow{3}{*}{ Entrada } & \multirow{3}{*}{$\begin{array}{l}\text { El diputado Ignacio } \\
\text { Urrutia (UDI) }\end{array}$} & $\begin{array}{l}\text { protagonizó un polémico } \\
\text { episodio }\end{array}$ & \\
\hline & & \multirow[t]{2}{*}{$\begin{array}{l}\text { lanzó un insulto contra el ex } \\
\text { Presidente Salvador Allende }\end{array}$} & $\begin{array}{l}\text { luego de que se solicitara un } \\
\text { minuto de sliencio en el } \\
\text { Congreso en recuerdo de las } \\
\text { víctimas de la dictadura }\end{array}$ \\
\hline & & & $\begin{array}{l}\text { al cumplirse } 39 \text { años del golpe de } \\
\text { Estado }\end{array}$ \\
\hline
\end{tabular}

Si nos quedáramos en un análisis que centre superficialmente la atención en el léxico y en el estilo utilizados, podríamos inferir que este TN corresponde a una FD de izquierda y que, por ello, realiza una recontextualización coorientada del TN de Co- 
operativa. Pero, si hacemos un análisis más profundo y relacionamos las formas lingüísticas en el archivo semántico de la formación y reconocemos su relación con el interdiscurso, llegamos a una conclusión diferente. Recordemos que no son las palabras y frases emitidas lo que inscribe a los discursos en una FD o en otra, sino el sentido que estas adquieren en un momento particular, en un proceso de significación continuo y determinado por tensiones y dislocaciones que otorgan diferentes valores a los signos. Este es el efecto de determinación del interdiscurso.

Sobre este supuesto, consideramos las expresiones golpe de Estado, dictadura y Salvador Allende, entre otras, como signos ideológicos (Voloshinov, 1992: 31-50) que tienen valores determinados en la historización discursiva. Dicho de otra manera, los textos no son producidos y puestos en circulación en un vacío social; al contrario, tienen sentido en tanto activan presupuestos que conforman una memoria discursiva. Para El Mercurio, golpe de Estado, dictadura y Salvador Allende son signos que no tienen el mismo valor que para los medios de la FD de izquierda. Las palabras pueden ser las mismas, pero el sentido no. En la FD de derecha, se presupone: El golpe de Estado fue necesario, la dictadura no fue tan sangrienta, Salvador Allende fue un mal presidente. Esa memoria fue modelada durante años por discursos como este:

\section{Editorial del domingo 11 de diciembre de 2006}

Pinochet y sus pares intervinieron frente a una crisis ya fuera de control. Esa intervención, no obstante su altísimo costo en cuanto a imagen y a incomprensión del mundo, evitó a Chile una guerra civil -que habría sido, sin duda alguna, muy sangrienta- o la caída en un totalitarismo.

Tras esas convulsiones, Pinochet condujo un gobierno -autoritario para sus partidarios, dictatorial para sus opositores- cuyo más perdurable legado fue dar a Chile un modelo de emprendimiento basado en la libertad individual y en el derecho de propiedad, valores crecientemente abandonados por décadas, y que en 1973 se hallaban en la imposibilidad de ejercerse, por la crítica situación del Estado de Derecho.

El Mercurio realizó un esfuerzo discursivo para disimular la colisión de matrices que sí quedó manifiesta en el discurso de COPESA. Si bien ha justificado siempre el golpe de Estado, en los últimos años ha expuesto una moderada condena a las violaciones de los derechos humanos perpetradas por el gobierno militar. Se suma a esto una postura conservadora que rechaza, en general, los agravios a las autoridades institucionales.

\subsection{Las recontextualizaciones coorientadas}

Como era previsible, todos los medios ubicados en la FD de izquierda mantuvieron los patrones estilísticos e ideológicos del TN de Cooperativa. A continuación, presentamos el análisis de The Clinic y El Mostrador.

\subsubsection{The Clinic}

En su página web, este semanario se limitó a resumir el TN de radio Cooperativa, manteniendo tanto la organización general de la información como el estilo. El título dado a la noticia fue: Diputado Ignacio Urrutia y minuto de silencio por Allende: " ¿A los cobardes que se suicidaron ese día también le vamos a rendir homenaje?”. 
Como se aprecia en la Tabla 4, el actor privilegiado en las principales cláusulas es el diputado de la UDI. No hubo mención de otros actores:

Tabla 4. Principales cláusulas del TN de The Clinic

\begin{tabular}{|c|c|c|c|}
\hline $\begin{array}{l}\text { Ubicación de la } \\
\text { cláusula }\end{array}$ & Actor & Acción & Circunstancias \\
\hline Título & $\begin{array}{l}\text { Diputado Ignacio } \\
\text { Urrutia }\end{array}$ & $\begin{array}{l}\text { [dijo] “¿A los cobardes que } \\
\text { se suicidaron ese día } \\
\text { también le vamos a rendir } \\
\text { homenaje?" }\end{array}$ & $\begin{array}{l}\text { [por/en relación con] minuto de } \\
\text { silencio a Allende }\end{array}$ \\
\hline \multirow{4}{*}{ Entrada } & \multirow{4}{*}{$\begin{array}{l}\text { El diputado } \\
\text { gremialista Ignacio } \\
\text { Urrutia }\end{array}$} & \multirow{3}{*}{ se mandó una de aquellas } & este martes \\
\hline & & & en el Congreso \\
\hline & & & luego que \\
\hline & & alegó & $\begin{array}{l}\text { por el minuto de silencio en honor a } \\
\text { Salvador Allende y a las víctimas del } \\
11 \text { de septiembre. }\end{array}$ \\
\hline
\end{tabular}

Las equivalencias son lineales y materializan una sola matriz, la misma que radio Cooperativa. La memoria discursiva activada acá asigna un valor determinado a los signos ideológicos Salvador Allende y 11 de septiembre, los cuales son de suma importancia para la FD de izquierda.

Puede llamar la atención que The Clinic haya suprimido en las primeras cláusulas las expresiones "dictadura" y golpe de Estado" (presentes en el TN de radio Cooperativa y en el de El Mercurio), pero su discurso también (re)produjo un sentido historizado. El mismo 11 de septiembre, The Clinic publicó el audio del último discurso de Salvador Allende y un informe que demostraba que el golpe de Estado fue impulsado por el gobierno de Estados Unidos. Los signos ideológicos Salvador Allende y 11 de septiembre están estrechamente vinculados a dictadura y golpe de Estado, los cuales, junto a otros signos, conforman la red de sentido presupuesta por el interdiscurso, mediante numerosas apariciones anteriores en textos noticiosos, informes y columnas de opinión. También publicó el spot de campaña de Ignacio Urrutia, titulado: Ignacio Urrutia en campaña: "Soy el diputado que Piñera necesita", vinculando el acto de agravio con la figura del entonces Presidente Piñera y con el gobierno en general. Este plexo de sentidos, conformado por información explícita e implícita, constituyó un encuadre que determinó una recontextualización coorientada fuerte.

El discurso de The Clinic materializó tres matrices de sentido. Una es la relativa a la crítica pública de los hombres con X cualidad. La otra es la referida a la validez de la democracia y, por ende, al rol del ejército. Podría ser formulada: La democracia no debe ser interrumpida por la fuerza. La tercera matriz es la típica de la categoría política: El gobierno debe administrar bien el Estado. En el interdiscurso de la FD de izquierda se presupone siempre que el gobierno de Allende fue positivo, que el golpe de Estado fue negativo y que el gobierno militar generó injusticias y atentó contra los derechos humanos. 


\subsubsection{El Mostrador}

Este diario realizó una recontextualización coorientada que está en un grado intermedio entre el modo fuerte y el débil. Por un lado, expresa un moderado rechazo al diputado Urrutia y, por otro, un marcado rechazo de la dictadura. Tal como ocurre con el TN de The Clinic, visto superficialmente, el TN de El Mostrador parece estar más próximo a la línea discursiva de La Tercera que El Mercurio.

Veamos la organización clausal de este texto:

Tabla 5. Principales cláusulas del TN de El Mostrador

\begin{tabular}{|c|c|c|c|}
\hline $\begin{array}{c}\text { Ubicación de la } \\
\text { cláusula }\end{array}$ & Actor & Acción & Circunstancias \\
\hline Título & $\begin{array}{l}\text { Ignacio Urrutia, el } \\
\text { diputado UDI }\end{array}$ & $\begin{array}{l}\text { no respetó minuto de silencio } \\
\text { en memoria de Allende y } \\
\text { víctimas de la dictadura }\end{array}$ & \\
\hline \multirow{3}{*}{ Bajada } & \multirow{3}{*}{ El parlamentario } & \multirow[b]{2}{*}{ Reaccionó } & con molestia \\
\hline & & & $\begin{array}{l}\text { a la petición de su par René } \\
\text { Alinco }\end{array}$ \\
\hline & & $\begin{array}{l}\text { se preguntó si había que } \\
\text { rendir un homenaje "al } \\
\text { cobarde que se suicidó ese } \\
\text { día" }\end{array}$ & $\begin{array}{l}\text { en alusión al fallecido ex } \\
\text { presidente socialista }\end{array}$ \\
\hline \multirow{4}{*}{ Entrada } & \multirow{4}{*}{$\begin{array}{l}\text { El diputado de la UDI } \\
\text { Ignacio Urrutia }\end{array}$} & \multirow{4}{*}{ no quiso sumarse } & a sus pares \\
\hline & & & en el hemiciclo de la Cámara \\
\hline & & & $\begin{array}{l}\text { cuando se concedió un minuto de } \\
\text { silencio en memoria del ex } \\
\text { presidente Salvador Allende y de } \\
\text { las miles de víctimas de la } \\
\text { dictadura de Augusto Pinochet }\end{array}$ \\
\hline & & & $\begin{array}{l}\text { al cumplirse este martes el } \\
\text { trigésimo noveno aniversario del } \\
\text { golpe militar de } 1973\end{array}$ \\
\hline
\end{tabular}

Se construyó un actor privilegiado, el diputado Urrutia, quien cargo con toda la responsabilidad de lo sucedido. La referencia hizo hincapié en su rol institucional. Las cuatro acciones predicadas guardan una relación equivalente entre sí. Son diferentes formas de decir lo mismo. No se mencionaron los otros actores (diputado Alinco, presidente de la Cámara), aunque sí se detallaron las acciones desarrolladas por estos.

Este TN desarrolló un segundo tema: la finalización de la investigación oficial sobre la muerte del ex Presidente Allende, decretada ese día por la Corte de Apelaciones de Santiago. Eliminó la referencia a la repercusión en las redes sociales, común a todos los TNs anteriores.

Ambos temas permitieron la materialización discursiva de las tres matrices de sentido que reconstruimos en el discurso de The Clinic y que propusimos como comunes a la FD de izquierda. La conducta de Urrutia fue utilizada como el disparador para reafirmar la crítica a la dictadura pinochetista, en un país donde ese pasado y su pro- 
yección sobre el presente todavía son cuestiones controversiales (Santander Molina, 2007; Mönckeberg, 2009; Garretón, M. y Garretón, A., 2010).

Este último análisis refuerza la coherencia de los procesos de recontextualización efectuados. Tanto en los medios de derecha como en los de izquierda, el discurso contextualizado obligó a la revisión y a la reafirmación de los límites que distinguen cada FD.

\section{Conclusiones}

La meta general de este trabajo fue presentar un modelo de análisis de las orientaciones y modos de la recontextualización periodística. Para ello, efectuamos una síntesis de aportes de tres científicos sociales que han explotado el concepto de sistema: $\mathrm{N}$. Luhmann, M. Pêcheux y B. Bernstein. Esto nos permitió describir y explicar las posibilidades que se abren cuando la representación discursiva de un acontecimiento es tomada por un medio particular como insumo para la producción de un discurso que siempre remite a una historia y a un juego de tensiones y diferencias.

Establecimos dos orientaciones básicas: la coorientación (u orientación positiva) y la contraorientación (u orientación negativa). Ambas, reflejan la afinidad existente entre la ideología expresada por el discurso a recontextualizar y la ideología expresada por el discurso recontextualizador. También propusimos dos modos básicos: fuerte y débil. Estas categorías deben ser entendidas en términos graduales y no lineales. También deben ser comprendidas en el marco de una perspectiva que recupere la historicidad y los presupuestos de todo discurso.

El análisis realizado nos permitió testear la utilidad heurística de este modelo y explicar los casos predecibles y los que, en principio, resultan extraños o, incluso, anómalos. Pudimos distinguir sentidos diferentes en textos noticiosos que, en apariencia, son similares y sentidos similares a textos noticiosos que, en apariencia, son diferentes.

De este modo, aspiramos a contribuir al desarrollo de los estudios críticos del discurso periodístico, en particular a aquellos que intentan desentrañar las regularidades que fundan las condiciones de posibilidad tanto de lo que se dice como de lo no se dice, pero igual se comunica.

\section{Referencias bibliográficas}

ALCÁNTARA SÁEZ, Manuel (2004): "La ideología de los partidos políticos latinoamericanos", en Working Papers Online Series, Departamento de Ciencia Política y Relaciones Internacionales, Universidad Autónoma de Madrid: http://portal.uam.es/portal/page/portal/UAM_ORGANIZATIVO/Departamentos/CienciaPoliticaRelacionesInternacionales/publicaciones $\% 20 \mathrm{en} \% 20 \mathrm{red} /$ working_papers/archivos/20_2004.pdf [fecha de consulta: 23/04/2013].

AVENDAÑO, Octavio (2011): "La oposición política en Chile durante el período 1990-2011. Una aproximación conceptual y empírica". CS, núm. 8, julio- diciembre 2011, pp. 147-186.

BERSNTEIN, Basil (1998): Pedagogía, control simbólico e identidad. Madrid, Morata. 
CASTILLO, Marcelo (2011): "En Chile la prensa es de derecha", entrevista publicada en diario Página 12: http://www.pagina12.com.ar/diario/elmundo/4-1770282011-09-18.html [Consulta: 20 de noviembre de 2013].

CORRALES JORQUERA, Osvaldo y SANDOVAL MOYA, Juan (2005): "Concentración del mercado de los medios, pluralismo, y libertad de expresión”. Centro de Estudios de la Comunicación, Universidad de Chile: http://www.comunicacion .uchile.cl/docs/corrales2005.pdf [Consulta: 10 de mayo de 2012].

DÁVILA, Mireya y FUENTES, Claudio (2003): Promesas de cambio. Izquierda y derecha en el Chile contemporáneo. Santiago, FLACSO.

DERMOTA, Ken (2002): Chile inédito: El periodismo bajo democracia. Santiago, Ediciones B.

GARRETÓN, Manuel y GARRETÓN, Roberto (2010): "La democracia incompleta en Chile: La realidad tras los ranckings internacionales". Revista de Ciencia Política, vol. 30, núm. 1: http://www.scielo.cl/pdf/revcipol/v30n1/art07.pdf [Consulta: 23 de mayo de 2012].

GONZÁLEZ RODRÍGUEZ, Gustavo (2012): "Medios de comunicación en Chile, bajo el signo de la concentración", versión traducida del cap. "The Media in Chile. The restoration of democracy and the subsquent concentration of media ownership", en Jairo Lugo-Ocando, The Media in Latin America. Londres, McGraw Hill, Londres, 2008: www.lapetus.uchile.cl/lapetus/c1/download.php?id=1218 [fecha de consulta: 13/05/2012].

LADRIÈRE, Jean (1978): El reto de la racionalidad. Salamanca, Sígueme-UNESCO.

LUHMANN, Niklas (1998): Sistemas sociales. Barcelona, Anthropos.

LUHMANN, Niklas (2000): La realidad de los medios de masas. Barcelona, Anthropos.

LUHMANN, Niklas (2006): La sociedad de la sociedad. México, Herder.

LUNA, Juan Pablo y ZECHMEISTER, Elizabeth (2010): Cultura politica de la democracia en Chile, 2010. Consolidación democrática en las Américas en tiempos dificiles. Vanderbilt University: http://www.vanderbilt.edu/lapop/chile/Chile-2010cultura-politica.pdf [Consulta: 14 de noviembre de 2012].

MERRILL, Jessica (2010): "La Libertad do Expression Politica en la Prensa Escrita de Chile". Independent Study Project (ISP) Collection, Paper 861: http://digitalcollections.sit.edu/isp_collection/861 [fecha de consulta: 04/03/2013].

MÖNCKEBERG, María Olivia (2009): Los magnates de la prensa. Santiago, Debate. PÊCHEUX, Michel (1978): Hacia el análisis automático del discurso. Madrid, Gredos.

PÊCHEUX, Michel (1990): L'inquietude du discours. Paris, De Cendres.

RAITER, Alejandro (2008): "Dominación y discurso", en RAITER, Alejandro y ZULLO, Julia: Lingüistica y política, $2^{\circ}$ ed., pp. 19-23. Buenos Aires, Biblos. 
SANTANDER MOLINA, Pedro (2007): "Medios en Chile 2002-2005: entre la lucha por el poder y la sumisión al espectáculo", en SANTANDER MOLINA, Pedro (ed.): Los medios en Chile: voces y contextos. Valparaíso, PUCV, pp. 11-38.

SUNKEL, Guillermo (2002): La prensa sensacionalista y los sectores populares. Bogotá, Norma.

SUNKEL, Guillermo y GEOFFROY, Esteban (2001): Concentración económica de los medios de comunicación. Santiago, LOM Ediciones.

VOLOSHINOV, Valentin (1992): El marxismo y la filosofía del lenguaje. Madrid, Alianza.

\section{Corpus de publicaciones digitales}

Cooperativa: "Diputado UDI Ignacio Urrutia calificó a Allende como 'el cobarde que se suicidó"', http://www.cooperativa.cl/noticias/pais/manifestaciones/11-de-septiembre/diputado-udi-ignacio-urrutia-califico-a-allende-como-el-cobarde-que-sesuicido/2012-09-11/125352.html

El Mercurio, editorial: “Augusto Pinochet Ugarte" (01/12/2006): http://diario.elmercurio.com/detalle/index.asp?id $=\{$ e236020b-fb4f-4a36-a717-a6c05f495468 $\}$

El Mercurio: "Diputado UDI lanzó insulto contra Allende en el Congreso": http://www.emol.com/noticias/nacional/2012/09/11/560026/diputado-udi-que-interrumpio-minuto-de-silencio-en-el-congreso-con-insulto-a-allende.html

El Mostrador: "Ignacio Urrutia, el diputado UDI que no respetó minuto de silencio en memoria de Allende y víctimas de la dictadura": http://www.elmostrador.cl/pais/2012/09/11/ignacio-urrutia-el-diputado-udi-queno-respeto-minuto-de-silencio-en-memoria-de-allende-y-victimas-de-la-dictadura/

La Cuarta: "Minuto de silencio a Allende prendió la mecha en el Congreso": http://www.lacuarta.com/noticias/cronica/2012/09/63-143823-9-homenaje-aallende-prendio-la-mecha-en-el-congreso.shtml

La Segunda: "Diputado UDI lanzó insulto contra Allende en el Congreso": $\mathrm{http} / / / \mathrm{www}$.lasegunda.com/movil/detallenoticia.aspx?idnoticia $=780348$

La Tercera: "Diputados UDI protagonizan incidente en la Cámara de Diputados": http://www.latercera.com/noticia/politica/2012/09/674-482904-9-diputados-udiprotagonizan-incidente-en-la-camara-en-minuto-de-silencio-por.shtml

The Clinic, "Diputado Ignacio Urrutia y minuto de silencio por Allende: '¿A los cobardes que se suicidaron ese día también le vamos a rendir homenaje?": http://www.theclinic.cl/2012/09/11/diputado-ignacio-urrutia-y-minuto-de-silenciopor-allende-a-los-cobardes-que-se-suicidaron-ese-dia-tambien-le-vamos-a-rendirhomenaje/ 